WUB/07-06

\title{
The Higgs mechanism as a cut-off effect
}

\author{
Nikos Irges ${ }^{1}$, Francesco Knechtli ${ }^{2}$ and Magdalena Luz $^{2}$ \\ 1. High Energy and Elementary Particle Physics Division \\ Department of Physics, University of Crete, 71003 Heraklion, Greece \\ e-mail: irges@physics.uoc.gr \\ 2. Fachbereich C, Bergische Universität Wuppertal \\ 42097 Wuppertal, Germany \\ e-mail: \\ knechtli@physik. uni-wuppertal.de, luz@physik.uni-wuppertal.de
}

\begin{abstract}
We compute the Coleman-Weinberg potential with a finite cut-off for pure $S U(2)$ and $S U(3)$ five-dimensional gauge theories compactified on an interval. We show that besides the expected Coulomb phase located at and in the vicinity of the free infrared stable or "trivial" fixed point, the theory possesses also a Higgs phase. We compare the results from the potential computation with lattice data from simulations.
\end{abstract}




\section{Introduction}

A five-dimensional pure $S U(N)$ gauge theory is "trivial". This remains true when one of the dimensions is compactified on a circle of radius $R$ or an interval. The simplest way to see this is to look at the two dimensionless quantities parameterizing the theory

$$
N_{5}=\pi R \Lambda \quad \text { and } \quad \beta=2 N /\left(g_{5}^{2} \Lambda\right)
$$

$N_{5}$ is the ratio of the cut-off $\Lambda$ over the compactification scale $1 /(\pi R)$ and the coupling $\beta$ is derived from the dimensionful five-dimensional gauge coupling $g_{5}$ (and is appropriate for a lattice cut-off as we will use in this work). The crucial observation is that at a critical value of the coupling $\beta=\beta_{c}$, a first order phase transition occurs [1,2, 3, 4]. For $\beta<\beta_{c}$ the system finds itself in a confined phase and for $\beta>\beta_{c}$ in a deconfined phase. The non-perturbative spectrum of a pure $S U(2)$ five-dimensional gauge theory compactified on an interval [5, 6] was determined by lattice simulations [7, 8]. The outcome is that the particle masses in lattice units are presumably too large in the confined phase in order to be "measured" in simulations. The situation is different in the deconfined phase, where we therefore expect to possibly reproduce the Higgs sector of the Standard Model. Because of these facts, removing the cut-off (i.e. taking the limit $\Lambda \rightarrow \infty$ ) in the deconfined phase, while keeping the dimensionless coupling $g_{5}^{2} \Lambda$ perturbative in the limit, is possible only at the trivial point $-g_{5} \rightarrow 0$ and $\beta \rightarrow \infty$ - of the phase diagram (the $N_{5}-\beta$ plane). For an

extra dimension of non-zero size 1 , in this limit, also $N_{5} \rightarrow \infty$. Perturbative computations performed with an infinite cut-off amount therefore to being located at the trivial point of the phase diagram, where interactions vanish. Any higher dimensional gauge theory is expected to have these generic properties [9].

More precisely, triviality can be understood by looking at the effective four-dimensional coupling

$$
g_{4}^{2}=\frac{g_{5}^{2}}{2 \pi R}=\frac{N}{N_{5} \beta}
$$

The evolution of this coupling with the scale is a calculation that requires a cut-off. This was done in [10], where the following 1-loop formula was found for one extra dimension

$$
\left(\beta N_{5}\right)(\Lambda)=\left(\beta N_{5}\right)(\mu)-b_{4} \ln (\Lambda / \mu)+b_{5}[(\Lambda / \mu)-1]
$$

expressed here in the dimensionless parameters using Eq. (1.2).$\mu$ is a reference scale such that $1 / R<\mu<\Lambda$ and $R$ is kept fixed. $b_{4}$ is a model dependent number and $b_{5}$ is also cut-off independent in the limit of large $\Lambda / \mu$. One immediately sees from Eq. (1.3) that as $\Lambda \rightarrow \infty$, the 1-loop corrected effective coupling $g_{4}$ goes to zero. For large $N_{5}$ and $\beta$

\footnotetext{
${ }^{1}$ We will avoid at all times the singular limit $R=0$.
} 
the formula describes physics in the vicinity of the trivial point, while its applicability becomes questionable as $N_{5} \beta \rightarrow 0$ where cut-off effects run out of control. Nevertheless it is legitimate to ask how much one can really lower the product $N_{5} \beta$ so that the theory can be safely described analytically while keeping $g_{4}^{2}$ perturbative.

Our goal is to study spontaneous symmetry breaking (SSB). Gauge coupling evolution is not the most appropriate tool, since SSB has to be put by hand in the $\beta$-function computation. Instead, a scalar potential is needed. In particular, one-loop computations of the Coleman-Weinberg potential [11] for compactified extra-dimensional gauge theories have been carried out at infinite cut-off aiming to explain the Higgs mechanism. According to this scenario, (some of) the fifth dimensional components of the gauge fields play the role of the Higgs field [12, 13, 14, 15, 16, 17, 18, 19]. Several interesting properties have emerged, some of them encouraging some of them not from a phenomenological point of view. The most impressive virtue is the finiteness of the Higgs mass, which is believed to hold non-perturbatively due to the non-local origin of the operator whose fluctuations are responsible for generating this mass. For the same reason it seems though impossible for the rank of the bulk gauge symmetry to be broken. To break the rank and/or to obtain reasonable phenomenology, additional assumptions have to be employed. One common feature of these models is the introduction of extra matter fields, as SSB seems not to be possible in the pure gauge theory. Recent works related to such issues include $[20,21,22,23,24,25,26,27,28,29,30,31,32,33,34,35,36,37,38$. In any case it seems rather hard to obtain naturally a reasonable hierarchy of masses in the sector which is supposed to reproduce physics in the bosonic sector of the Standard Model.

In this work we intend to show that in the interior of the $N_{5^{-}} \beta$ phase diagram where the cut-off is finite, there is a transition into a broken rank phase of the five-dimensional pure $S U(N)$ gauge theory. The tool that allows to see this is the (not necessarily perturbative in $\beta$ ) expansion in the cut-off

$$
-\mathcal{L}=\frac{1}{2 g_{5}^{2}} \operatorname{tr}\{F \cdot F\}+\sum_{p_{i}} c^{\left(p_{i}\right)}\left(N_{5}, \beta\right) a^{p_{i}-4} \mathcal{O}^{\left(p_{i}\right)}+\ldots
$$

of the effective lagrangean [39,40,41,42], where $F$ is the field strength, $\mathcal{O}^{\left(p_{i}\right)}$ is an operator 2 of dimension $p_{i}>4$ and $c^{\left(p_{i}\right)}\left(N_{5}, \beta\right)$ is in general a non-perturbative function of $\beta$ and $N_{5}$ in the interior of the phase diagram. The sum runs over all independent operators of dimension $p_{i}$. The regularization assumed for this action is one where space-time is Euclidean and discrete (i.e. $N_{5}$ is an integer) and the cut-off is related to the lattice spacing $a$ as $\Lambda=1 / a$. The various operators appearing in $\mathcal{L}$ contribute to the masses of the states that make up the spectrum, in case some fields acquire a vacuum expectation

\footnotetext{
${ }^{2}$ Additional boundary counterterms appear when the theory is defined on an interval. Their significance for our discussion will become clear in the following.
} 
value (vev). The scalar potential at one-loop can be computed by inserting the mass matrix into the Coleman-Weinberg formula.

We expect that there is a region in the parameter space around the trivial point where the effective lagrangean Eq. (1.4) describes well the theory, in the sense that a truncation of the expansion is meaningful. It will turn out to be a good approximation also when we will compare with results from lattice simulations.

To begin, computing the Coleman-Weinberg potential with a cut-off should move us somewhere in the interior of the phase diagram. Moreover, we will seek regions of the phase diagram where a reasonable truncation of the expansion Eq. (1.4) is possible. The reason is that in such a case, the theory can be described analytically and nonperturbatively to a good approximation with a finite number of parameters. In a lattice regularization, the leading order value of the coefficients can be read off by expanding the Wilson plaquette action to appropriate order, while the general $c^{\left(p_{i}\right)}\left(N_{5}, \beta\right)$ can be either computed in perturbation theory or "measured" by means of a numerical simulation. We will not determine these coefficients here. Instead we use them as variable parameters of our potential calculation and compare its results with the lattice data.

\section{The Coleman-Weinberg potential}

\subsection{Review of the continuum calculation and a few comments}

Let us first remove the cut-off. The calculation in this case can be carried out in the continuum without having to refer explicitly to the lattice regulator. The ColemanWeinberg scalar potential $V$ in $D$ dimensions is defined at one-loop by

$$
\int[\mathrm{D} \phi] e^{-S_{E}} \sim \mathrm{e}^{-V} \equiv \frac{1}{\sqrt{\operatorname{det}\left[\square+M^{2}\right]}} .
$$

This one-loop approximation is guaranteed to be satisfactory as long as we keep $g_{4} \ll 1$. The potential $V$ can be extracted as follows:

$$
V=-\frac{1}{2} \frac{\pi^{D / 2}}{(2 \pi)^{D}} \int_{0}^{\infty} \frac{\mathrm{d} t}{t^{\frac{D+2}{2}}} \operatorname{tr}\left\{\mathrm{e}^{-t M^{2}}\right\} .
$$

In order to specify the mass spectrum, one expands the fields à la Kaluza-Klein (KK), where the eigenvalues of the mass matrix $M$ are of the form $m_{n}(\alpha)=(n+f(\alpha)) / R$, $n \in \mathbb{Z}$. In our case the shifts $f(\alpha)$ of the KK numbers are due to some of the components of the gauge field $A_{5}$ taking a vacuum expectation value (vev).

To be more specific, we will consider an $S U(2)$ gauge theory in five dimensions compactified on an interval of size $\pi R$, which is equivalent to an $S^{1} / \mathbb{Z}_{2}$ orbifold. The gauge 
field $A_{M}=-i g_{5} A_{M}^{B} T^{B}\left(T^{B}=\sigma^{B} / 2, B=1,2,3\right.$ are the $S U(2)$ generators) is defined on the circle $S^{1}$ along the extra dimension. Its components $A_{M}^{B}$ are divided into even and odd under the orbifold parity, which is the product of the parity under Euclidean reflection ( +1 for $A_{\mu}, \mu=0,1,2,3$ and -1 for $\left.A_{5}\right)$ with the parity under group conjugation $(+1$ for the components with $B=3$ and -1 for $B=1,2)$. The conjugation matrix is $g=-i \sigma_{3}$. The even $E$ and odd $O$ fields are expanded in the KK basis as

$$
\begin{aligned}
& E\left(x, x_{5}\right)=\frac{1}{\sqrt{2 \pi R}} E^{(0)}(x)+\frac{1}{\sqrt{\pi R}} \sum_{n=1}^{\infty} E^{(n)}(x) \cos \left(n x_{5} / R\right), \\
& O\left(x, x_{5}\right)=\frac{1}{\sqrt{\pi R}} \sum_{n=1}^{\infty} O^{(n)}(x) \sin \left(n x_{5} / R\right) .
\end{aligned}
$$

On the boundary the even fields that survive the orbifold projection are the scalar (from a four-dimensional point of view) components $A_{5}^{1,2}$, which will be our complex Higgs field, and the gauge boson component $A_{\mu}^{3}$, which we will call the $Z^{0}$ gauge boson.

The mass-squared terms in the lagrangean $\mathcal{L}$ for the gauge bosons $A_{\mu}$ are in the continuum

$$
\left(\bar{D}_{5} A_{\mu}^{A}\right)\left(\bar{D}_{5} A_{\mu}^{A}\right)
$$

where $\bar{D}_{5} A_{M}^{A}=\partial_{5} A_{M}^{A}+g_{5} f^{A B C}\left\langle A_{5}^{B}\right\rangle A_{M}^{C}$. The masses of the KK modes of $A_{\mu}$ are obtained by expanding the field components as in Eq. (2.3) and Eq. (2.4). The mass-squared terms for the scalars $A_{5}$ originate from the gauge-fixing term in the lagrangean and are

$$
\frac{1}{\xi}\left(\bar{D}_{5} A_{5}^{A}\right)\left(\bar{D}_{5} A_{5}^{A}\right) \text {. }
$$

We will work in the gauge $\xi=1$. In order to find the mass eigenvalues, the gauge symmetry can be used to allow only one component of $A_{5}$ to take a vev, here for example

$$
\left\langle A_{5}^{1}\right\rangle=\frac{v}{\sqrt{2 \pi R}}, \quad\left\langle A_{5}^{2}\right\rangle=\left\langle A_{5}^{3}\right\rangle=0 .
$$

The mass matrix for the four-dimensional fields is diagonal in the KK index $n$. The eigenvalue shifts for $n \neq 0$ are $f(\alpha)=0, \pm \alpha$ and are the same for the gauge bosons and the scalars. The parameter $\alpha$ is defined as

$$
\alpha=\frac{g_{5} v R}{\sqrt{2 \pi R}}
$$

The mass of the "Cartan" component is $m_{Z^{0}}=\alpha / R$ and the the masses of the scalar zero-modes are $0, \alpha / R$. The potential Eq. (2.2) has the simple 3 periodic form [18, 19]

$$
V=-\frac{3 \cdot P}{64 \pi^{6} R^{4}} \sum_{m=1}^{\infty} \frac{\cos (2 \pi m \alpha)}{m^{5}},
$$

\footnotetext{
${ }^{3}$ The derivation of Eq. (2.9) involves a Poisson resummation of the KK index. Thereafter a divergent contribution $m=0$ is dropped since it does not depend on the parameter $\alpha$.
} 
where $P=3$ is the multiplicity of states ( 2 from physical degrees of polarization of $A_{\mu}$, 1 from $A_{5}$ ). Succinctly expressed, the dimensionless modulus in Eq. (2.8) acquired a non-trivial potential at the quantum level by the Hosotani mechanism [13, 14].

The fast way to see why the rank can not break at infinite cut-off is to look at the commutator of the vacuum expectation value of the Polyakov line around the extra dimension (which is the physical meaning of $A_{5}$ ) with the generators of the Cartan subalgebra:

$$
\left[e^{-i \pi \alpha \sigma^{1}}, H_{i}\right]
$$

which clearly vanishes for $\alpha \in \mathbb{Z}$. From the point of view of the potential, it is obvious from Eq. (2.9) that the term that determines the true vacuum is the first term in the expansion in $m$, proportional to $\cos (2 \pi \alpha)$. Clearly, Eq. (2.9) then has a global minimum at $\alpha=\alpha_{\min }=0 \bmod \mathbb{Z}$ as a result of which the KK tower shifts by an integer and thus SSB of the rank of the gauge group does not occur, consistently with the symmetry argument.

Nevertheless, a non-trivial Higgs mass is implied by $V$. In fact, the Higgs mass formula found by computing the second derivative of the potential Eq. (2.9) at the minimum agrees with the one obtained by a direct continuum perturbative calculation of vacuum polarization diagrams in dimensional regularization [43, 44]

$$
\left.\left(m_{H} R\right)_{\text {pert }}^{2} \equiv R^{2} \frac{\mathrm{d}^{2} V}{\mathrm{~d} v^{2}}\right|_{\alpha=\alpha_{\min }}=\left.\frac{9 \zeta(3)}{8 \pi^{4}} \frac{1}{N_{5} \beta}\right|_{\alpha=0}
$$

expressed in terms of the dimensionless parameters 4 We are particularly interested in the ratio

$$
\rho_{H Z^{0}}=\frac{m_{H}}{m_{Z^{0}}}
$$

whose one-loop value is naturally undetermined at the trivial point.

To summarize, at the trivial point, the $Z^{0}$ gauge boson remains massless and the Higgs is inclined towards masslessness as well. These phenomenological obstacles are expected to persist generically as long as the theory is probed in the close vicinity of the trivial point.

It is important therefore to investigate if these are properties valid throughout the entire phase diagram. For instance, in order that the rank is preserved non-perturbatively, a discrete global symmetry must act on $\alpha$ so that it can protect the perturbative minimum of the potential. We see no such symmetry. In fact, a careful lattice simulation study of an $S U(2)$ orbifold gauge theory, for a range of finite values of $N_{5}$ and $\beta$ shows that there is no leftover massless $U(1)$ gauge boson, suggesting a non-perturbative breaking of the

\footnotetext{
${ }^{4}$ Note that the squared Higgs mass on the orbifold is just one half of that on the circle [45].
} 
rank [7,8]. As a simple plausibility argument, let us assume that $\alpha$, not being protected by any symmetry, changes from its integer value at the trivial point by a cut-off dependent shift $\epsilon(\Lambda)$ in the interior of the phase diagram. Then,

$$
\left[e^{-i \pi(1-\epsilon(\Lambda)) \sigma^{1}}, H_{i}\right] \sim \mathcal{O}(\epsilon(\Lambda))
$$

the Polyakov loop does not commute anymore with the $H_{i}$, allowing for SSB of the rank, which can occur even if $\epsilon(\Lambda)$ is infinitesimal.

As we will show in the remaining of this letter, the transition from the Coulomb into the Higgs phase on the circle is sharp with the modulus $\alpha$ shifting discretely due to the presence of cut-off dependent higher dimensional operators. On the orbifold, the minimum is further shifted continuously due to boundary counterterms, which also bring important cut-off effects.

\subsection{Cut-off effects in the mass formulae}

As a first step, we would like to derive the first non-trivial correction in the effective action which determines the leading correction to the mass formula. For simplicity we consider the theory regulated on an infinite lattice. The $S U(N)$ Wilson plaquette action is

$$
S_{\mathrm{W}}=-\frac{\beta}{2 N} \sum_{z} \sum_{M, N} \operatorname{tr}\left\{2-U_{M N}(z)-U_{M N}^{\dagger}(z)\right\}
$$

where $z=\left(x_{\mu}, x_{5}\right)$ and $\mu=1, \ldots, 4, M=1, \ldots, 5$. The plaquettes are defined as $U_{M N}(z)=U(z, M) U(z+a \hat{M}, N) U^{-1}(z+a \hat{N}, M) U^{-1}(z, N)$ and a link is related to the gauge field via $U(z, M)=e^{a A_{M}(z)}$. Using the Baker-Campbell-Hausdorff formula, given the Lie algebra elements $X, Y, P, Q$ one can determine the $F_{i}$ such that

$$
e^{a X} e^{a Y} e^{a P} e^{a Q}=e^{\sum_{i} a^{i} F_{i}}
$$

where $i$ runs from 1 to some specified order $I$, with all the $F_{i}$ anti-hermitian. This implies that in expanding the plaquette action with $X=A_{M}(z), Y=A_{N}(z+a \hat{M})$, $P=-A_{M}(z+a \hat{N})$ and $Q=-A_{N}(z)$, the non-zero contributions arrange themselves as

$$
S_{\mathrm{W}}=\frac{\beta}{2 N} \sum_{n=1}^{\infty} \frac{2}{(2 n) !}\left(\sum_{i=1}^{I} a^{i} F_{i}\right)^{2 n} .
$$

Let us start from the $n=1$ term. This is $\left(a F_{1}+a^{2} F_{2}+\ldots\right)^{2}$, whose leading order part, by substituting the explicit forms

$$
F_{1}=A_{M}(z)+A_{N}(z+a \hat{M})-A_{M}(z+a \hat{N})-A_{N}(z), \quad F_{2}=\left[A_{M}(z), A_{N}(z)\right]
$$


is recognized to correspond to the dimension 4 operator $\mathcal{O}^{(4)}=\sum_{M, N} \operatorname{tr}\left\{F_{M N} F_{M N}\right\}$. The next terms in the expansion generate the dimension 6 operator [46]

$$
\mathcal{O}^{(6)}=\sum_{M, N} \frac{1}{24} \operatorname{tr}\left\{F_{M N}\left(D_{M}^{2}+D_{N}^{2}\right) F_{M N}\right\}
$$

and so forth. The squared gauge boson masses (when $A_{\mu}$ is expanded in the KK basis) can be extracted by diagonalizing the operator

$$
\bar{D}_{5} \bar{D}_{5}+\frac{a^{2}}{12}\left(\bar{D}_{5} \bar{D}_{5}\right)^{2}+\ldots,
$$

where $\bar{D}_{5}=\partial_{5}+\left[\left\langle A_{5}\right\rangle, \cdot\right]$. The mass squared matrix is therefore itself an expansion

$$
M^{2}=\left(M^{2}\right)^{(4)}+\frac{a^{2}}{12}\left(M^{2}\right)^{(6)}+\ldots, \quad\left(M^{2}\right)^{(6)}=\left(\left(M^{2}\right)^{(4)}\right)^{2}
$$

with the superscript denoting the dimension of the contributing operator. As mentioned, non-perturbatively, the coefficient $1 / 12$ in Eq. (2.19) should be replaced by a generic coefficient

$$
c \equiv c^{(6)}\left(N_{5}, \beta\right) .
$$

The masses of the field $A_{5}$ come entirely from the gauge fixing term and do not receive corrections from the action.

On the orbifold, an additional contribution to the mass matrix comes from the boundary counterterm

$$
\mathcal{L}^{\text {bound. }}=\frac{\pi a c_{0}}{4} F_{5 \mu}^{\hat{a}} F_{5 \mu}^{\hat{a}}\left[\delta\left(x_{5}\right)+\delta\left(x_{5}-\pi R\right)\right]
$$

in the effective lagrangean Eq. (1.4). It will give a contribution only to the mass matrix of the even gauge fields $A_{\mu}^{a}$ because these do not vanish on the boundaries. We note that the counterterm Eq. (2.22) does not appear at one-loop in perturbation theory [43,44] but is expected to arise at higher orders [5]. The boundary coefficient should also be understood as a cut-off dependent function $c_{0}\left(N_{5}, \beta\right)$ at a generic point of the phase diagram.

It is straightforward to obtain the cut-off corrected eigenvalues of the mass matrices. We will consider two models. One is the $S U(2)$ model we have already described and the other is its $S U(3)$ generalization. In this case the orbifold breaks the symmetry down to $S U(2) \times U(1)$ on the boundaries. The even fields are $A_{\mu}^{1,2,3,8}$ and $A_{5}^{4,5,6,7}$, the rest of them are odd. The vev can be aligned along $\left\langle A_{5}^{4}\right\rangle=v / \sqrt{2 \pi R}$ and the eigenvalues are again parameterized by $\alpha$ defined in Eq. (2.8).

For the $S U(2) \stackrel{\mathbb{Z}_{2}}{\rightarrow} U(1)$ model the non-zero mode eigenvalues of the mass matrix $(M R)^{2}$ are (we recall the relation $\pi R=N_{5} a$ )

$$
\begin{aligned}
\left(m_{n} R\right)^{2}= & n^{2}, \\
& (n \pm \alpha)^{2}+\frac{c_{0} \alpha^{2}}{2} \frac{\pi}{N_{5}}+c(n \pm \alpha)^{4} \frac{\pi^{2}}{N_{5}^{2}} .
\end{aligned}
$$


The single zero mode eigenvalue $\left(m_{Z^{0}} R\right)^{2}$ can be obtained by putting $n=0$ in Eq. (2.24). For the $S U(3) \stackrel{\mathbb{Z}_{2}}{\rightarrow} S U(2) \times U(1)$ model in the non-zero mode sector we find $(2 \times$ denotes degeneracies)

$$
\begin{aligned}
\left(m_{n} R\right)^{2}= & 2 \times n^{2}, \\
& (n \pm \alpha)^{2}+\frac{c_{0} \alpha^{2}}{4} \frac{\pi}{N_{5}}+c(n \pm \alpha)^{4} \frac{\pi^{2}}{N_{5}^{2}} \\
& 2 \times\left((n \pm \alpha / 2)^{2}+\frac{c_{0}(\alpha / 2)^{2}}{4} \frac{\pi}{N_{5}}+c(n \pm \alpha / 2)^{4} \frac{\pi^{2}}{N_{5}^{2}}\right) .
\end{aligned}
$$

In the zero-mode sector there is a single zero eigenvalue (corresponding to $\left(m_{\gamma} R\right)^{2}$ ), one eigenvalue which is obtained by putting $n=0$ in Eq. (2.26) (corresponding to $\left.\left(m_{Z^{0}} R\right)^{2}\right)$ and a pair of eigenvalues which is obtained by putting $n=0$ in Eq. (2.27) (corresponding to $\left.\left(m_{W^{ \pm}} R\right)^{2}\right)$. The only subtle step in these calculations is that for the boundary contributions we have kept only their $n$-independent parts and dropped $\mathrm{O}(1 / n)$ corrections, which seems to be a satisfactory approximation.

Another new input immediately appears because of the finite cut-off. Namely, the Higgs vev should not exceed $1 / a$. This translates in the constraint

$$
|\alpha|<\sqrt{\frac{N N_{5}}{\pi^{2} \beta}}
$$

and the potential we are about to compute strictly makes sense only in this regime.

\subsection{Coleman-Weinberg potential with a cut-off}

We consider a one-component scalar field of mass $m$ on a $D$ dimensional Euclidean lattice with lattice spacing $a$. The effective potential can be written in the same form as in the continuum Eq. (2.2)

$$
V=-\frac{1}{2} \int_{\epsilon}^{\infty} \frac{\mathrm{d} t}{t} \operatorname{tr}\left[\mathrm{e}^{-t\left(m^{2}+\hat{p}^{2}\right)}\right]
$$

but here $\hat{p}_{\mu}=\frac{2}{a} \sin \left(\frac{a p_{\mu}}{2}\right)$ are the lattice momenta and the $p_{\mu}$ take values restricted to the interval $-\pi / a$ and $\pi / a$. Hence Eq. (2.29) is short for

$$
V=-\frac{1}{2} \int_{0}^{\infty} \frac{\mathrm{d} l}{l} \mathrm{e}^{-\frac{1}{l}\left(m^{2} a^{2}+2 D\right)} \frac{1}{a^{D}} \mathrm{I}_{0}^{D}\left(\frac{2}{l}\right),
$$

where we have substituted $1 / t$ by $l / a^{2}$ and $\mathrm{I}_{0}(2 / l)$ is the $0^{\text {th }}$ modified Bessel function. The total potential $V$ is the sum over all mass states of the ghosts, gauge and scalar particles. 


\subsubsection{The $S U(2)$ case}

Let us start with the gauge bosons $A_{\mu}$. We include only the $n$ dependent terms of the mass eigenvalues of Eq. (2.24) into $m_{n}$. This leads to the modified KK masses

$$
m_{n}^{2}=\left(\frac{n+\alpha}{R}\right)^{2}+c a^{2}\left(\frac{n+\alpha}{R}\right)^{4} .
$$

The boundary correction is accounted for by a shift in the exponential in Eq. (2.30), i.e. (setting $D=4$ )

$$
e^{-\frac{1}{l}\left(m_{n}^{2} a^{2}+8\right)} \rightarrow e^{-\frac{1}{l}\left(m_{n}^{2} a^{2}+8+c_{0} \frac{a^{3} \alpha^{2}}{2 R^{3}}\right)} .
$$

The $c_{0}$ term is of $O(a)$ relative to the mass squared and is therefore expected to be small, such that it can be expanded. At the same time, we use an expansion in $c$ throwing away all terms of $O\left(a^{3}\right)$ or higher in the joint expansion. Schematically, we will collect the terms

$$
V=\underbrace{f_{0}}_{O(1)}+\underbrace{f_{2} c}_{O\left(a^{2}\right)}+\underbrace{f_{1} c_{0}}_{O(a)}+\underbrace{\tilde{f}_{2} c_{0}^{2}}_{O\left(a^{2}\right)} .
$$

We start with the pure bulk contributions, that is $f_{0}$ and $f_{2}$. On the lattice the values of $n$ are restricted to the interval $n=0,1, . ., N_{5}-1$ but we extend them to $n \rightarrow \infty$ for the calculation. This is justified because the higher modes are expected to contribute very little [10]. Hence, summing over all KK states gives

$$
V^{\text {bulk }}=-\frac{1}{2 a^{4}} \sum_{n \in \mathbb{Z}} \int_{0}^{\infty} \frac{\mathrm{d} l}{l} e^{-\frac{1}{l}\left(m_{n}^{2} a^{2}+8\right)} I_{0}^{4}\left(\frac{2}{l}\right) .
$$

Note that on the orbifold the extension to the sum over $n \in \mathbb{Z}$ is achieved by combining the $+\alpha$ and $-\alpha$ contributions in the eigenvalues Eq. (2.24). We expand in the $O\left(a^{2}\right)$ correction to the mass formula Eq. (2.31) and obtain

$$
V^{\text {bulk }}=-\frac{1}{2 a^{4}} \sum_{k=0}^{\infty} c^{k} \frac{\left(-a^{4}\right)^{k}}{k ! R^{4 k}} \int_{0}^{\infty} \frac{\mathrm{d} l}{l} \frac{1}{l^{k}} e^{-\frac{8}{l}} \mathrm{I}_{0}^{4}\left(\frac{2}{l}\right) \sum_{r=0}^{4 k}\left(\begin{array}{c}
4 k \\
r
\end{array}\right) \alpha^{4 k-r} \sum_{n \in \mathbb{Z}} e^{-\left(\frac{n+\alpha}{R}\right)^{2} \frac{a^{2}}{l}} n^{r},
$$

where we have expressed $(n+\alpha)^{4 k}$ through the binomial identity. The next step is a Poisson resummation. From the ordinary Poisson resummation formula follows the identity of its derivatives ( $A$ and $b$ are constants and $m$ an integer not to be confused with the mass $m$ )

$$
\frac{1}{\pi^{r}} \frac{\partial^{r}}{\partial b^{r}} \sum_{n} e^{-\left(\pi A n^{2}+\pi b n\right)}=\frac{1}{\pi^{r}} \frac{\partial^{r}}{\partial b^{r}} \sqrt{A}^{-1} \sum_{m} e^{-\frac{\pi}{A}(m+i b / 2)^{2}} .
$$

The $\sum_{n}$ appears in Eq. (2.36) exactly in the same way as in the rhs of Eq. (2.35), hence we need to find the resummation of the exponential alone and then take its $r$-th derivative. We define functions $f^{(r, m)}(\alpha, l)$ by the relation

$$
f^{(r, m)}(\alpha, l): \frac{\partial^{r}}{\partial b^{r}} e^{-\frac{\pi}{A}\left(m+i \frac{b}{2}\right)^{2}}=f^{(r, m)}(\alpha, l) e^{-\frac{\pi}{A}\left(m+i \frac{b}{2}\right)^{2}} .
$$


These functions have the property that $f^{(r,-m)}(\alpha, m)=\left[f^{(r, m)}(\alpha, m)\right]^{*}$. Thus $V^{\text {bulk }}$ can be written as

$$
V^{\text {bulk }}=-\frac{1}{2} \frac{R}{a} \sum_{k}(-1)^{k} c^{k} V_{k}
$$

where

$$
\begin{aligned}
V_{k}= & \frac{a^{4(k-1)}}{k ! R^{4 k}} \sum_{r=0}^{4 k} \frac{1}{\pi^{r-\frac{1}{2}}}\left(\begin{array}{c}
4 k \\
r
\end{array}\right) \alpha^{4 k-r} \int_{0}^{\infty} \mathrm{d} l l^{-\frac{1}{2}-k} e^{-\frac{8}{l}} I_{0}^{4}\left(\frac{2}{l}\right) \times \\
& \times\left(f^{(r, 0)}(\alpha, l)+\sum_{m>0} 2 \operatorname{Re}\left[f^{(r, m)}(\alpha, l) e^{-2 i \pi m \alpha}\right] e^{-\frac{\pi^{2} R^{2} l m^{2}}{a^{2}}}\right) .
\end{aligned}
$$

To be consistent in the order of the expansion we must truncate this sum at $k=1$. A fact that one should notice here and keep in mind is that the $k=1$ term comes with the opposite sign with respect to the $k=0$ term.

The original integral in Eq. (2.30) has a logarithmic divergence for $l \rightarrow \infty$. The Poisson resummation regularizes the integral in the sense that after the resummation it is finite up to a divergent term coming from $m=0$. This term is constant in $\alpha$ and can be neglected. Hence the terms corresponding to $f_{0}$ and $f_{2}$ are

$$
V_{0}=\frac{2 \sqrt{\pi}}{a^{4}} \int_{0}^{\infty} \frac{\mathrm{d} l}{\sqrt{l}} e^{-\frac{8}{l}} \mathrm{I}_{0}^{4}\left(\frac{2}{l}\right) \sum_{m>0} \cos (2 \pi m \alpha) e^{-N_{5}^{2} m^{2} l}
$$

and

$$
V_{1}=\frac{2 \sqrt{\pi}}{a^{4}} \int \mathrm{d} l \sqrt{l} e^{-\frac{8}{l}} \mathrm{I}_{0}^{4}\left(\frac{2}{l}\right) \sum_{m>0} \cos (2 \pi m \alpha)\left(N_{5}^{4} m^{4} l^{2}-3 N_{5}^{2} m^{2} l+\frac{3}{4}\right) e^{-N_{5}^{2} m^{2} l}
$$

respectively, where we used $R \pi=N_{5} a$. For the boundary potential $V^{\text {bound. }}$ determined by the terms $f_{1}$ and $\tilde{f}_{2}$ we need the first and second order terms in $c_{0}$ at $c=0$. After a Poisson resummation, they look very similar to $V_{0}$ in Eq. (2.40). Apart from a prefactor, the only difference is the modified power of $l$ in the integrand.

Summing up, we finally obtain $V^{\text {gauge }}=V^{\text {bulk }}+V^{\text {bound. }}$, or explicitly

$$
\begin{aligned}
V^{\text {gauge }} & =-\frac{1}{2} \frac{N_{5}}{\pi}\left(V_{0}-c V_{1}\right)+ \\
& +\frac{c_{0}}{4} \frac{\alpha^{2} \pi^{5 / 2}}{N_{5}^{2}} \int_{0}^{\infty} \mathrm{d} l l^{-3 / 2} e^{-\frac{8}{l}} \mathrm{I}_{0}^{4}\left(\frac{2}{l}\right)\left(1+2 \sum_{m>0} \cos (2 \pi m \alpha) e^{-N_{5}^{2} l m^{2}}\right) \\
& -\frac{c_{0}^{2}}{16} \frac{\alpha^{4} \pi^{11 / 2}}{N_{5}^{5}} \int_{0}^{\infty} \mathrm{d} l l^{-5 / 2} e^{-\frac{8}{l}} \mathrm{I}_{0}^{4}\left(\frac{2}{l}\right)\left(1+2 \sum_{m>0} \cos (2 \pi m \alpha) e^{-N_{5}^{2} l m^{2}}\right)
\end{aligned}
$$

per gauge field $A_{\mu}$.

Note that in the boundary corrections the $m=0$ term can not be dropped because it is $\alpha$-dependent. However, the higher negative power in $l$ makes it converge as $l \rightarrow \infty$. On 
the other hand, the $c_{0}^{2}$ correction in Eq. (2.42) diverges in the limit $l \rightarrow 0$. This can be dealt with by splitting the corresponding integral in two domains. For small $l$ one can use Eq. (2.34) directly, with $m_{n}^{2}$ set to $(n+\alpha)^{2} / R^{2}$ in which case the only potential problems arise when $n+\alpha=0$. This however is not a real problem because on one hand at $\alpha=0$ the $c_{0}$ corrections vanish identically and on the other $\alpha$ will be always smaller than one due to the constraint in Eq. (2.28). For large $l$ one can then use Eq. (2.42).

The mass matrices for $A_{5}$ and the Faddeev-Popov ghosts are identica 5 and generated through $\bar{D}_{5}^{2}$ only. Moreover they do not get cut-off corrections from the bulk and boundary action. The contribution to the potential for $A_{5}$ is

$$
V^{\text {scalar }}=\left.V^{\text {gauge }}\right|_{c_{0}=c=0} \text {. }
$$

In the notation of Eq. (2.1) the 1-loop path integral for our theory is

$$
\frac{\operatorname{det}\left[\prod_{n}\left(-\partial_{\mu} \partial_{\mu}+m_{n, \text { scalar }}^{2}\right)\right]}{\operatorname{det}\left[\prod_{n}\left(-\partial_{\mu} \partial_{\mu}+m_{n, \text { gauge }}^{2}\right)\right]^{D / 2} \operatorname{det}\left[\prod_{n}\left(-\partial_{\mu} \partial_{\mu}+m_{n, \text { scalar }}^{2}\right)\right]^{1 / 2}}
$$

where $m_{n \text {,gauge }}$ is given by Eq. (2.24) and $m_{n \text {,scalar }}=\left.m_{n \text {,gauge }}\right|_{c_{0}=c=0}$. For $D=4$ this is equivalent to a Coleman-Weinberg potential

$$
V=4 V^{\text {gauge }}+V^{\text {scalar }}-2 V^{\text {scalar }}
$$

where the last (negative) contribution comes from the ghosts.

\subsubsection{The $S U(3)$ case}

The eigenvalues of the $S U(3)$ mass matrix Eq. (2.26) and Eq. (2.27) are very similar to those of the $S U(2)$ model in Eq. (2.24). They can be obtained by shifting $c_{0} \rightarrow c_{0} / 2$ and for $W$ in addition by setting $\alpha \rightarrow \alpha / 2$. Otherwise the derivation of the potential is exactly the same as in the $S U(2)$ case. We obtain for the $Z^{0}$

$$
\begin{aligned}
V_{Z^{0}}^{\text {gauge }} & =-\frac{1}{2} \frac{N_{5}}{\pi}\left(V_{0}-c V_{1}\right) \\
& +\frac{c_{0}}{8} \frac{\alpha^{2} \pi^{5 / 2}}{a^{4} N_{5}^{2}} \int \mathrm{d} l l^{-3 / 2} e^{-8 / l} \mathrm{I}_{0}^{4}\left(\frac{2}{l}\right)\left(1+2 \sum_{m>0} \cos (2 \pi m \alpha) e^{-N_{5}^{2} m^{2} l}\right) \\
& -\frac{c_{0}^{2}}{64} \frac{\alpha^{4} \pi^{11 / 2}}{a^{4} N_{5}^{5}} \int \mathrm{d} l l^{-5 / 2} e^{-8 / l} \mathrm{I}_{0}^{4}\left(\frac{2}{l}\right)\left(1+2 \sum_{m>0} \cos (2 \pi m \alpha) e^{-N_{5}^{2} m^{2} l}\right)
\end{aligned}
$$

with $V_{0}$ and $V_{1}$ as in Eqs. (2.40) and (2.41). Scalar and ghost contributions are exactly the same as for $S U(2)$ because they do not see the $c_{0}$ corrections anyway. In the case of

\footnotetext{
${ }^{5}$ Only the orbifold parities are opposite. Those of the ghosts are equal to the ones of the gauge bosons, as it follows by considering their interaction term in the lagrangean.
} 
the $W$ bosons the two different parts of the gauge bulk contributions are

$$
V_{0}^{W^{ \pm}}=\frac{2 \sqrt{\pi}}{a^{4}} \int \frac{\mathrm{d} l}{\sqrt{l}} e^{-8 / l} \mathrm{I}_{0}^{4}\left(\frac{2}{l}\right) \sum_{m>0} \cos (\pi m \alpha) e^{-N_{5}^{2} m^{2} l}
$$

and

$$
V_{1}^{W^{ \pm}}=\frac{2 \sqrt{\pi}}{a^{4}} \int \mathrm{d} l \sqrt{l} e^{-8 / l} \mathrm{I}_{0}^{4}\left(\frac{2}{l}\right) \sum_{m>0} \cos (\pi m \alpha)\left(N_{5}^{4} m^{4} l^{2}-3 N_{5}^{2} m^{2} l+\frac{3}{4}\right) e^{-N_{5}^{2} m^{2} l} .
$$

Finally, adding the boundary corrections leads to

$$
\begin{aligned}
V_{W^{ \pm}}^{\text {gauge }} & =-\frac{1}{2} \frac{N_{5}}{\pi}\left[V_{0}^{W^{ \pm}}-c V_{1}^{W^{ \pm}}\right] \\
& +\frac{c_{0}}{32} \frac{\alpha^{2} \pi^{5 / 2}}{a^{4} N_{5}^{2}} \int \mathrm{d} l l^{-3 / 2} e^{-8 / l} \mathrm{I}_{0}^{4}\left(\frac{2}{l}\right)\left(1+2 \sum_{m>0} \cos (\pi m \alpha) e^{-N_{5}^{2} m^{2} l}\right) \\
& -\frac{c_{0}^{2}}{1024} \frac{\alpha^{4} \pi^{11 / 2}}{a^{4} N_{5}^{5}} \int \mathrm{d} l l^{-5 / 2} e^{-8 / l} \mathrm{I}_{0}^{4}\left(\frac{2}{l}\right)\left(1+2 \sum_{m>0} \cos (\pi m \alpha) e^{-N_{5}^{2} m^{2} l}\right) .
\end{aligned}
$$

Naturally, here the scalar/ghost contributions are also modified accordingly, that is we have to replace $\cos (2 \pi m \alpha)$ by $\cos (\pi m \alpha)$ in Eq. (2.43). Summing up, the total ColemanWeinberg potential for $S U(3)$ is

$$
V=4 V_{Z^{0}}^{\text {gauge }}+8 V_{W^{ \pm}}^{\text {gauge }}-2 V_{W^{ \pm}}^{\text {scalar }}-V^{\text {scalar }}
$$

The mode counting includes an additional factor of 2 for the degenerate $W$ bosons.

\section{$3 \quad$ Numerical results}

Fig. 1 shows the Coleman-Weinberg potential for $S U(2)$, Eq. (2.45), choosing $N_{5}=6$, $c=13.0$ and $c_{0}=0.0121$. We plot the dimensionless product $R^{4} V$. From the structure of Eq. (2.42) we can see that on the circle (where $c_{0}=0$ ) for large enough $c$, the term $c V_{1}$ dominates over $V_{0}$ and there is a discrete shift of the minimum from $\alpha=0$ to $\alpha=1 / 2$. For $N_{5}=6$, if $c$ is set to its tree-level value $1 / 12$ in the Wilson plaquette effective action, there is no SSB. The critical value is at $c \approx 1.72$. Given the non-renormalizability of the theory, there are quantum corrections which are power-like in the cut-off and large discrepancies from the tree level value are perhaps not unexpected. That is on $S^{1}$, the transition from the Coulomb into the Higgs phase is sharp and the minimum shifts by half an integer. On $S^{1} / \mathbb{Z}_{2}$ (where $c_{0} \neq 0$ ) the non-trivial minimum can drift continuously away from $\alpha=1 / 2$ due to the $\alpha$-dependent prefactors in $V^{\text {bound. }}$. In general, for a given $c$ there is a maximal value of $c_{0}$ for which there is SSB, that is the potential has a minimum at $0<\alpha_{\min }<1$. For $c_{0}$ larger than this value the minimum is at $\alpha=0$. These expectations are confirmed 


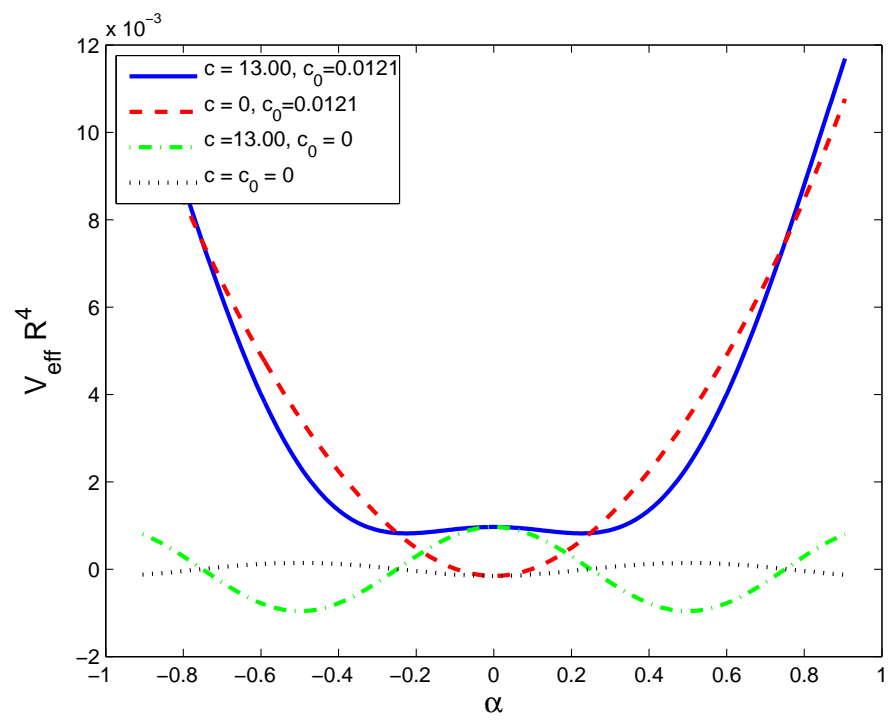

Figure 1: The Coleman-Weinberg potential for $S U(2)$ at $N_{5}=6$. The plot shows four different cases: the potential with and without boundary and bulk corrections. The parameters are $c=13.0$ and $c_{0}=0.0121$, if not set to zero. The minimum for the full potential (solid line) lies at $\alpha_{\min }=0.225$.

by Fig. 1, which also shows that it is not possible to have SSB due to boundary effects only, that is with $c_{0}>0$ and $c=0$.

The masses of the $Z^{0}$ boson and its first excited state $Z^{0 *}$ correspond to the two lightest modes in Eq. (2.24) in the $S U(2)$ model. They depend on the value of $\alpha_{\min }$ which minimizes the potential in Eq. (2.45). We have

$$
m_{Z^{0}} R=\min _{n \in\{0,1\}} \sqrt{\left(n-\alpha_{\min }\right)^{2}+\frac{c_{0} \pi}{2 N_{5}} \alpha_{\min }^{2}+\frac{c \pi^{2}}{N_{5}^{2}}\left(n-\alpha_{\min }\right)^{4}}
$$

and the mass of the first excited state $m_{Z^{0 *}}$ corresponds to the $\max _{n \in\{0,1\}}$ in the above formula. In Fig. 2 we compare the analytical formulae Eq. (3.1) for $N_{5}=6, c=13.0$ and $c_{0}=0.0121$ (solid lines) with the lattice results. We denote the number of points in the lattice along the spatial directions by $L / a$, along the time direction by $T / a$ and along the extra dimension by $N_{5}$ with $N_{5} \leq L / a$. We always compare results from the analytical formulae and the lattice with the same $N_{5}$. Increasing $L / a$ with $N_{5}$ fixed corresponds then to compactifying the extra dimension on the lattice.

The black symbols are our numerical data for $N_{5}=6, L / a=12$ and $T / a=96$. The points at $\alpha_{\min } \approx 0.25$ correspond to small $\beta$ values near the phase transition. On the lattice we define the Higgs field $\Phi$ by

$$
\Phi=\left[a A_{5}, g\right], \quad a A_{5}=\frac{1}{4 N_{5}}\left(P-P^{\dagger}\right),
$$




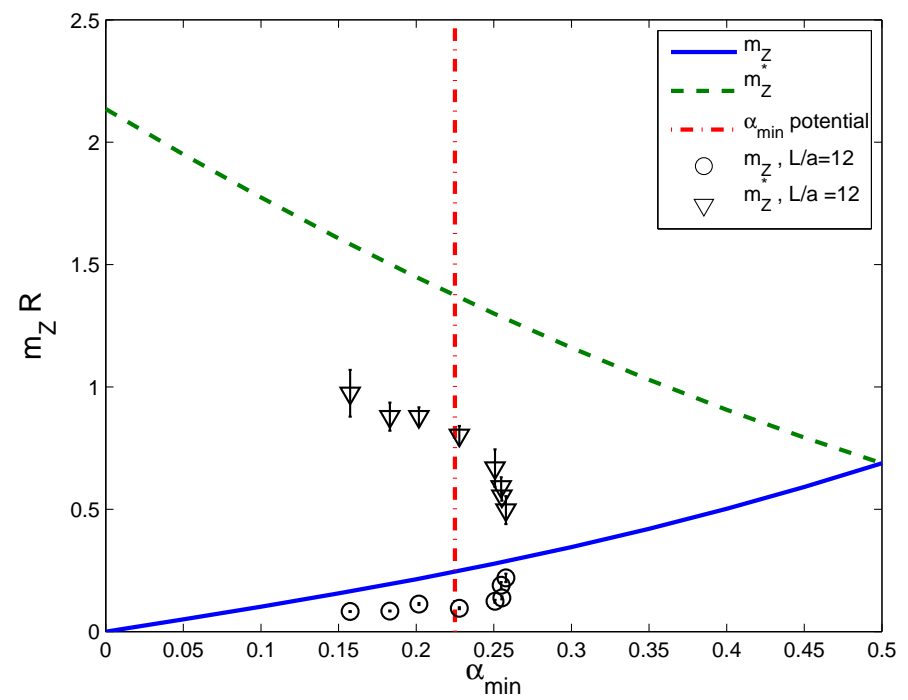

Figure 2: $m_{Z}$ and $m_{Z}^{*}$ as function of $\alpha_{\min }$ for $S U(2)$ at $N_{5}=6$. Comparison of the analytical formula Eq. (3.1) with the lattice data at $L / a=12$ and $T / a=96$.

where $g$ is the orbifold projection matrix and $P$ the Polyakov line 6 . We measure $\left\langle\operatorname{tr}\left\{\Phi \Phi^{\dagger}\right\}\right\rangle=$ $2 a^{2} g_{5}^{2}\left\langle A_{5}^{1}\right\rangle^{2}$. From Eq. (2.7) and Eq. (2.8) it follows that this observable can be expressed through $\alpha_{\min }$ as

$$
\left\langle\operatorname{tr}\left\{\Phi \Phi^{\dagger}\right\}\right\rangle=2 \alpha_{\min }^{2} \frac{\pi}{N_{5}^{2}} .
$$

If we take the values of $\left\langle\operatorname{tr}\left\{\Phi \Phi^{\dagger}\right\}\right\rangle$ from lattice simulations, Eq. (3.3) can be understood as an implicit function $\alpha_{\min }(\beta)$ for a given orbifold geometry. For $N_{5}=6, L / a=12$ and $T / a=96$ we plot this function in Fig. 3. We can then take the $Z$ and $Z^{*}$ masses from the same simulations and plot them in Fig. 2, The agreement with the analytical formula Eq. (3.1) is not so bad.

The Coleman-Weinberg potential has a minimum at $\alpha_{\min }=0.225$ for the chosen $c$ and $c_{0}$. This value is marked by the dash-dotted vertical line in Fig. 2, In our comparison we are taking the "bare" values of $\alpha_{\min }$ to be the same in the potential and in the lattice computations. There might be a finite renormalization factor relating them.

From the Coleman-Weinberg potential we can derive the Higgs mass by taking the second derivative, cf. Eq. (2.11). For a general gauge group $S U(N)$ it is

$$
\left(m_{H} R\right)^{2}=\left.\frac{N}{N_{5} \beta} R^{4} \frac{\mathrm{d}^{2} V}{\mathrm{~d} \alpha^{2}}\right|_{\alpha=\alpha_{\min }},
$$

where we used $g_{5}^{2} /(2 \pi R)=N /\left(N_{5} \beta\right)$. The second derivative is evaluated numerically at

\footnotetext{
6 We take the product of the gauge links along the extra dimension with no displacement and no smearing. For details on the lattice construction and the measurement of its observables, see [8].
} 


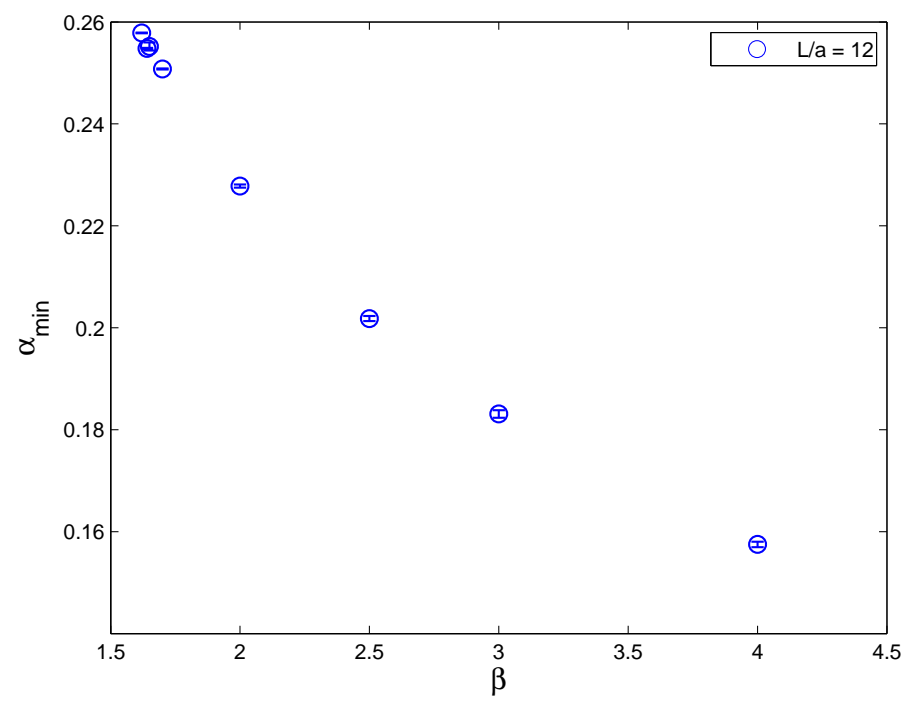

Figure 3: The function $\alpha_{\min }(\beta)$ for $S U(2)$ at $N_{5}=6$ determined from Eq. (3.3) and lattice simulations with $L / a=12$ and $T / a=96$.

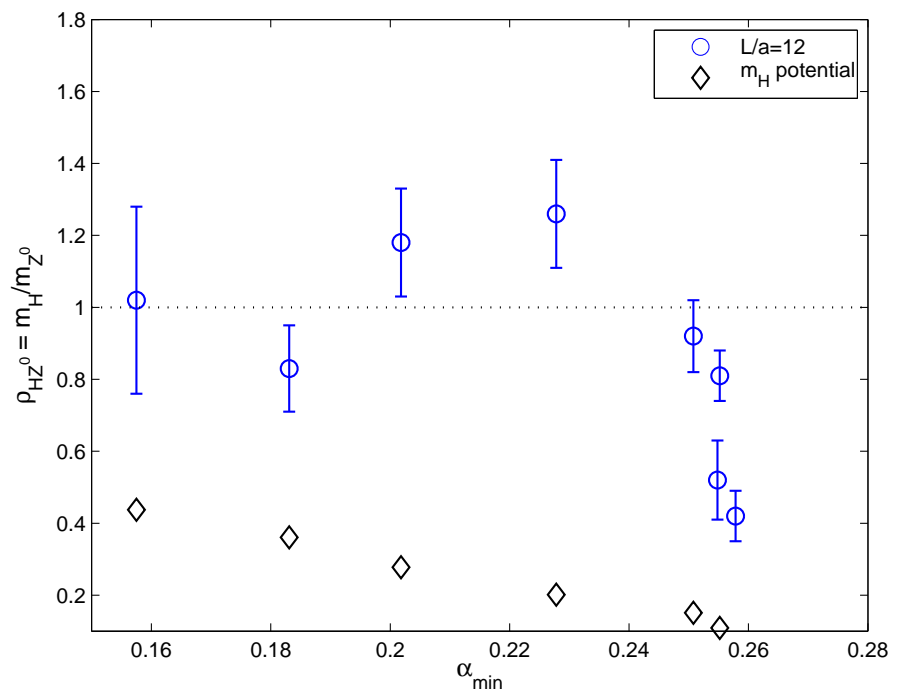

Figure 4: Ratio of the Higgs and the $Z^{0}$ boson masses for $S U(2)$ at $N_{5}=6$. Comparison of the lattice data with the Coleman-Weinberg potential computation.

the minimum $\alpha=\alpha_{\text {min }}$. Using the $Z^{0}$ mass from Eq. (3.1) we obtain values for the ratio $\rho_{H Z^{0}}=m_{H} / m_{Z^{0}}$. In Fig. 4 we compare these values with the lattice data at $N_{5}=6$. The parameters $c$ and $c_{0}$ in the Coleman-Weinberg potential are tuned such that the position of the minimum $\alpha_{\min }$ matches the value of the lattice data for $L / a=12$, see Fig. 3, and the corresponding value of $\beta$ is inserted in Eq. (3.4). The ratio $\rho_{H Z^{0}}$ computed in the lattice 


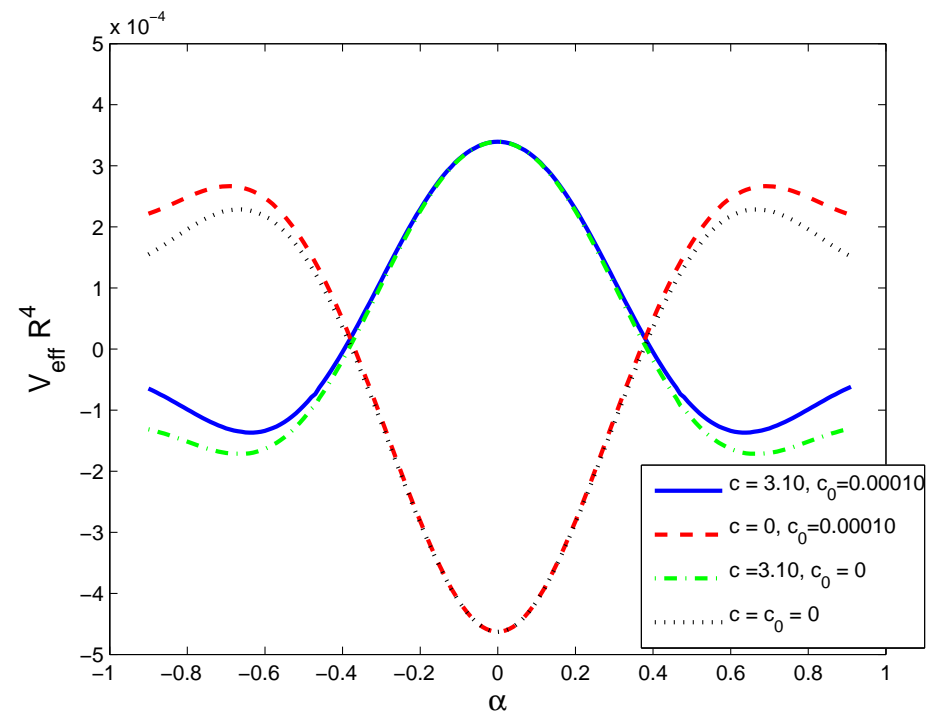

Figure 5: The Coleman-Weinberg potential for $S U(3)$ at $N_{5}=6$. The minimum for $c=3.1$ and $c_{0}=0.0001$ is at $\alpha_{\min }=0.64$.

simulations is significantly larger than the one computed with the Coleman-Weinberg potential. This is to be attributed to the values of the Higgs mass extracted from the potential, which are too small. On the lattice it is possible to get values of $\rho_{H Z^{0}} \geq 1$.

Finally, we consider the $S U(3)$ case. Here since we do not have lattice data yet, we will try to fit the analytical predictions to the experimental data. In Fig. 5 we plot the Coleman-Weinberg potential again in the dimensionless form $V R^{4}$ for $N_{5}=6, c=3.10$ and $c_{0}=0.0001$. The masses of the $Z^{0}$ and $W^{ \pm}$bosons are given by

$$
\begin{aligned}
& m_{Z^{0}} R=\min _{n \in\{0,1\}} \sqrt{\left(n-\alpha_{\min }\right)^{2}+\frac{c_{0} \pi}{4 N_{5}} \alpha_{\min }^{2}+\frac{c \pi^{2}}{N_{5}^{2}}\left(n-\alpha_{\min }\right)^{4}} \\
& m_{W} R=\min _{n \in\{0,1\}} \sqrt{\left(n-\frac{\alpha_{\min }}{2}\right)^{2}+\frac{c_{0} \pi}{4 N_{5}}\left(\frac{\alpha_{\min }}{2}\right)^{2}+\frac{c \pi^{2}}{N_{5}^{2}}\left(n-\frac{\alpha_{\min }}{2}\right)^{4}}
\end{aligned}
$$

(the choice of $n$ that maximizes the expressions gives the mass of the excited states). It is easy to see that for $\alpha_{\text {min }}>0.5$ the $Z^{0}$ mass corresponds to $n=1 \mathrm{in} \mathrm{Eq.} \mathrm{(3.5).} \mathrm{The} W$ mass is always given by $n=0$ in Eq. (3.6). Therefore the cosine of the Weinberg angle

$$
\cos \left(\theta_{\mathrm{W}}\right)=\frac{m_{W}}{m_{Z^{0}}}
$$

gets larger than the value $1 / 2$ which is the predicted value from the mass eigenvalues for $c=c_{0}=0$. The experimental value of the cosine of the Weinberg angle is 


\begin{tabular}{lllllll}
\hline$N_{5}$ & $c$ & $c_{0}$ & $m_{H} R$ & $\rho_{H Z^{0}}$ & $\cos \left(\theta_{\mathrm{W}}\right)$ & $\alpha_{\min }$ \\
\hline 2 & 3.1 & 0.02 & 0.206 & 0.54 & 0.899 & 0.647 \\
2 & 50.0 & 0.2 & 0.828 & 1.25 & 0.888 & 0.655 \\
3 & 3.1 & 0.0036 & 0.095 & 0.23 & 0.868 & 0.641 \\
3 & 50.0 & 0.05 & 0.400 & 0.43 & 0.836 & 0.645 \\
4 & 3.1 & 0.0007 & 0.053 & 0.12 & 0.877 & 0.645 \\
4 & 50.0 & 0.01 & 0.238 & 0.21 & 0.883 & 0.652 \\
6 & 3.1 & 0.0001 & 0.021 & 0.04 & 0.820 & 0.638 \\
6 & 50.0 & 0.0017 & 0.123 & 0.07 & 0.888 & 0.653 \\
\hline
\end{tabular}

Table 1: Results from the computation of the Coleman-Weinberg potential at small values of $N_{5}$. The Higgs mass is obtained from Eq. (3.4) setting $\beta=4.50$.

$\cos \left(\theta_{\mathrm{W}}^{\text {exp. }}\right) \simeq 0.877$ and can in principle be reached if the minimization of the potential gives the appropriate value of $\alpha_{\min }$, which turns out to be close to 0.65. Our general observation for $N_{5}=4,6$ or higher is that if we choose $c$ and $c_{0}$ such that the Weinberg angle is close to the experimental value, the Higgs mas:7 computed from Eq. (3.4) is always much smaller then the $Z^{0}$ mass, which is not phenomenologically acceptable. For example for the parameters as in Fig. 5 we get $\cos \left(\theta_{\mathrm{W}}\right)=0.82$ but $\rho_{H Z^{0}}=0.04$. In order to get larger values of $\rho_{H Z^{0}}$ we have to lower $N_{5}$, as it is demonstrated by the data in Table 1.

In fact for $N_{5}=2, c=50$ and $c_{0}=0.2$ we get $\rho_{H Z^{0}}=1.25$ which is the current lower limit from direct searches for the Higgs boson. At this value of $N_{5}$ cut-off effects become too large, the bound Eq. (2.28) gives $\alpha<0.37$. Nevertheless this is an indication that anisotropic lattices are needed to probe the small $N_{5}$ region, where we might expect phenomenologically relevant results.

\footnotetext{
${ }^{7}$ At one-loop order the Coleman-Weinberg potential does not depend on the gauge coupling but the Higgs mass, as defined in Eq. (3.4), does. For the $S U(3)$ pure gauge theory in five dimensions, the phase transition with periodic boundary conditions is at $\beta_{c}=4.35(15)$ [2]. When we quote a Higgs mass value for $S U(3)$ from Eq. (3.4) we set $\beta=4.50$.
} 


\section{Conclusions}

Calculations of the Coleman-Weinberg potential for five-dimensional gauge theories performed with an infinite cut-off yield the result of absence of spontaneous symmetry breaking (SSB). Therefore the presence of fermions is usually advocated.

In this work we have shown that SSB is observed in the pure gauge theory when an explicit cut-off is introduced. The lattice was our choice, since it is a gauge-invariant cut-off and we can compare the analytic results to simulation data. The analytic results were produced using a Symanzik effective langrangean, which is an expansion in operators of higher dimension accompanied by powers of the lattice spacing. A truncation to the leading order corrections is sufficient to produce evidence for SSB. In Fig. 2 we show the non-perturbative spectrum of gauge bosons for the $S U(2)$ theory compactified on an interval, as measured by lattice simulations, and compare it with the Coleman-Weinberg calculation. These results justify our approach. The presence of a Higgs phase with a massive $U(1)$ gauge boson is expected from considerations based on dimensional reduction. The Higgs boson in this theory has $U(1)$ charge equal to 2 [8]. The four-dimensional Abelian Higgs model for this charge has a phase separation between the confined and the Higgs phase [47].

We computed the Coleman-Weinberg potential for the phenomenologically more relevant case of gauge group $S U(3)$ broken into $S U(2) \times U(1)$ by the orbifold boundary conditions. We can reproduce the experimental value of the Weinberg angle and a ratio of the Higgs mass over the $Z$ boson mass larger than the current lower bound only if we lower the size $N_{5}$ of the extra dimension in lattice units, which points at the use of anisotropic lattices.

Small $N_{5}$ with a fixed cut-off corresponds to a small $R$ and dimensional reduction is expected to occur via compactification. This would be the regime where traditionally one would expect to see the SM. However, the large $N_{5}$ regime, which for a fixed cut-off corresponds to a large extra dimension could also be interesting (large $N_{5}$ at fixed $R$ with large cut-off drives us to the trivial point and we know what happens there) 8 This would require some localization mechanism 9 to work and as argued in [8] such a situation seems to have chances to be realized near the phase transition (small $\beta$ ). In addition, the towers of excited states collapse on their respective ground states [49] pushing the KK expansion outside its domain of validity, implying that the Coleman-Weinberg formula valid when the participating fields correspond to point particles, should not be trusted. In fact, the Coleman-Weinberg potential due to the local excitations is expected to be

\footnotetext{
${ }^{8}$ On the lattice this would correspond to choosing $N_{5}=L / a$.

${ }^{9}$ A prominent example is Ref. [48]
} 
suppressed, see also the discussion in [50]. In this part of the phase diagram the global Polyakov loop — nature of the Higgs and gauge bosons [8] can not be ignored. Beyond the lattice very few systematic analytical approaches are known that can probe the theory in this, inherently non-perturbative domain. The mean-field approximation could be one of them [6].

Acknowledgment. We would like to thank Philippe de Forcrand, Gero von Gersdorff and Peter Weisz for helpful discussions. The computer time for the lattice simulations has been kindly provided by the Swiss National Supercomputing Centre (CSCS) in Manno, by the Institute for Theoretical Physics of the University of Berne and by the University of Wuppertal.

\section{References}

[1] M. Creutz, Phys. Rev. Lett. 43 (1979) 553.

[2] B.B. Beard et al., Nucl. Phys. Proc. Suppl. 63 (1998) 775, hep-lat/9709120.

[3] S. Ejiri, J. Kubo and M. Murata, Phys. Rev. D62 (2000) 105025, hep-ph/0006217.

[4] K. Farakos, P. de Forcrand, C.P. Korthals Altes, M. Laine and M. Vettorazzo, Nucl. Phys. B655 (2003) 170, hep-ph/0207343.

[5] N. Irges and F. Knechtli, Nucl. Phys. B719 (2005) 121, hep-lat/0411018.

[6] F. Knechtli, B. Bunk and N. Irges, PoS LAT2005 (2006) 280, hep-lat/0509071.

[7] N. Irges and F. Knechtli, (2006), hep-lat/0604006.

[8] N. Irges and F. Knechtli, Nucl. Phys. B775 (2007) 283, hep-lat/0609045.

[9] J. Zinn-Justin, Quantum Field Theory and Critical Phenomena, 4th ed. (International Series of Monographs on Physics - Vol. 113, Clarendon Press, Oxford, 2002).

[10] K.R. Dienes, E. Dudas and T. Gherghetta, Nucl. Phys. B537 (1999) 47, hepph/9806292.

[11] S.R. Coleman and E. Weinberg, Phys. Rev. D7 (1973) 1888.

[12] P. Forgacs and N.S. Manton, Commun. Math. Phys. 72 (1980) 15.

[13] Y. Hosotani, Phys. Lett. B126 (1983) 309. 
[14] Y. Hosotani, Ann. Phys. 190 (1989) 233.

[15] Y. Sakamura and Y. Hosotani, Phys. Lett. B645 (2007) 442, hep-ph/0607236.

[16] Y. Hosotani, (2007), arXiv:0704.0883 [hep-ph].

[17] Y. Hosotani and Y. Sakamura, (2007), hep-ph/0703212.

[18] I. Antoniadis, K. Benakli and M. Quiros, New J. Phys. 3 (2001) 20, hep-th/0108005.

[19] M. Kubo, C.S. Lim and H. Yamashita, Mod. Phys. Lett. A17 (2002) 2249, hep$\mathrm{ph} / 0111327$.

[20] C. Csaki, C. Grojean and H. Murayama, Phys. Rev. D67 (2003) 085012, hep$\mathrm{ph} / 0210133$.

[21] N. Haba, M. Harada, Y. Hosotani and Y. Kawamura, Nucl. Phys. B657 (2003) 169, hep-ph/0212035.

[22] F. del Aguila, M. Perez-Victoria and J. Santiago, JHEP 02 (2003) 051, hepth/0302023.

[23] C.A. Scrucca, M. Serone and L. Silvestrini, Nucl. Phys. B669 (2003) 128, hep$\mathrm{ph} / 0304220$.

[24] C. Csaki, C. Grojean, H. Murayama, L. Pilo and J. Terning, Phys. Rev. D69 (2004) 055006, hep-ph/0305237.

[25] C. Biggio and M. Quiros, Nucl. Phys. B703 (2004) 199, hep-ph/0407348.

[26] G. Panico and M. Serone, JHEP 05 (2005) 024, hep-ph/0502255.

[27] G. Cacciapaglia, C. Csaki and S.C. Park, JHEP 03 (2006) 099, hep-ph/0510366.

[28] G. Panico, M. Serone and A. Wulzer, Nucl. Phys. B739 (2006) 186, hep-ph/0510373.

[29] N. Maru and K. Takenaga, Phys. Rev. D74 (2006) 015017, hep-ph/0606139.

[30] C. Grojean and G. Servant, Phys. Rev. D75 (2007) 043507, hep-ph/0607107.

[31] C.S. Lim and N. Maru, (2007), hep-ph/0703017.

[32] G.F. Giudice, C. Grojean, A. Pomarol and R. Rattazzi, (2007), hep-ph/0703164.

[33] K. Agashe, C. Csaki, C. Grojean and M. Reece, (2007), arXiv:0704.1821 [hep-ph].

[34] Y. Sakamura, (2007), arXiv:0705.1334 [hep-ph]. 
[35] G. von Gersdorff, (2007), arXiv:0705.2410 [hep-th].

[36] R. Barbieri, B. Bellazzini, V.S. Rychkov and A. Varagnolo, (2007), arXiv:0706.0432 [hep-ph].

[37] A.D. Medina, N.R. Shah and C.E.M. Wagner, (2007), arXiv:0706.1281 [hep-ph].

[38] C.S. Lim and N. Maru, (2007), arXiv:0706.1397 [hep-ph].

[39] K. Symanzik, Mathematical Problems in Theoretical Physics, eds. R. Schrader et al., Lecture Notes in Physics 153 (1982) 47, Presented at 6th Int. Conf. on Mathematical Physics, Berlin, West Germany, Aug 11-21, 1981.

[40] K. Symanzik, Nucl. Phys. B226 (1983) 187.

[41] K. Symanzik, Nucl. Phys. B226 (1983) 205.

[42] M. Lüscher, (1998), hep-lat/9802029.

[43] G. von Gersdorff, N. Irges and M. Quiros, Nucl. Phys. B635 (2002) 127, hepth/0204223.

[44] H.C. Cheng, K.T. Matchev and M. Schmaltz, Phys. Rev. D66 (2002) 036005, hep$\mathrm{ph} / 0204342$.

[45] M. Puchwein and Z. Kunszt, Annals Phys. 311 (2004) 288, hep-th/0309069.

[46] G.P. Lepage, (1996), hep-lat/9607076.

[47] E.H. Fradkin and S.H. Shenker, Phys. Rev. D19 (1979) 3682.

[48] G.R. Dvali and M.A. Shifman, Phys. Lett. B396 (1997) 64, hep-th/9612128.

[49] M. Laine, H.B. Meyer, K. Rummukainen and M. Shaposhnikov, JHEP 04 (2004) 027, hep-ph/0404058.

[50] D. Green, A. Lawrence, J. McGreevy, D.R. Morrison and E. Silverstein, (2007), arXiv:0705.0550 [hep-th]. 